\title{
底泥就地稳定化中零价铁 $\left(\mathrm{Fe}^{0}\right)$ 对有机污染物的作用及其对上覆水 体水质的影响
}

\author{
章 萍 $^{1}$, 相明雪 ${ }^{1}$, 马若男 ${ }^{1}$, 刘 强 ${ }^{1}$, 葛 刚 $^{1}$, 钱光人 ${ }^{2}$ \\ ( 1 : 南昌大学资源环境与化学工程学院, 鄱阳湖环境与资源利用教育部重点实验室, 南昌 330031) \\ (2: 上海大学环境与化学工程学院,上海 200444)
}

\begin{abstract}
摘 要: 在实验室模拟静态湖泊体系条件下, 通过向实际底泥中投加零价铁 $\left(\mathrm{Fe}^{0}\right)$, 考察反应前后底泥中有机物数量、种 类、总有机质含量以及上覆水体溶解氧 ( DO) 浓度、氧化还原电位 $(\mathrm{Eh}) 、 \mathrm{pH}$ 和化学需氧量 $\left(\mathrm{COD}_{\mathrm{Cr}}\right)$ 浓度等指标的变化, 探 讨 $\mathrm{Fe}^{0}$ 对底泥有机物的降解效果以及对上覆水体水质的影响. 结果表明, 投加 $\mathrm{Fe}^{0}$ 处理 $80 \mathrm{~d}$ 后, (1) 经 GC-MS 检测出底泥中 易被降解的小分子有机物 (分子量小于 200) 数量明显增多, 底泥总有机去除率为 $44 \%$. (2)上覆水体的 DO 浓度、Eh 和 $\mathrm{pH}$ 都有不同程度的变化. DO 浓度从 $6.6 \mathrm{mg} / \mathrm{L}$ 迅速下降至 $0.2 \mathrm{mg} / \mathrm{L}$, Eh 从 $150 \mathrm{mV}$ 左右下降至 $74 \mathrm{mV}, \mathrm{pH}$ 升高至 8.4 , 此体系 易形成厌氧环境; 且上覆水体中 $\mathrm{COD}_{\mathrm{Cr}}$ 低于纯底泥一水体系, 上覆水中 $\mathrm{DO}$ 浓度、 $\mathrm{Eh}$ 及 $\mathrm{pH}$ 与 $\mathrm{COD}_{\mathrm{Cr}}$ 浓度具有一定相关性. 综上,底泥中投加 $\mathrm{Fe}^{0}$ 可有效降解有机物, 且不会对上覆水体产生持久、较大的影响.
\end{abstract}

关键词: 就地稳定; 零价铁 $\left(\mathrm{Fe}^{0}\right)$; 底泥; 有机污染物; 上覆水

\section{Effect of zero-valent iron on organic pollutants in sediment and the related overlying water during in-situ treatment}

\author{
ZHANG Ping ${ }^{1}$, XIANG Mingxue ${ }^{1}$, MA Ruonan ${ }^{1}$, LIU Qiang ${ }^{1}$, GE Gang ${ }^{1}$ \& QIAN Guangren ${ }^{2}$ \\ (1: Key Laboratory of Poyang Lake Environment and Resource Utilization, Ministry of Education, School of Resources Envi- \\ ronmental \& Chemical Engineering, Nanchang University, Nanchang 330031, P.R. China) \\ (2: College of Environmental and Chemical Engineering, Shanghai University, Shanghai 200444, P.R. China)
}

\begin{abstract}
Based on the laboratory simulates a static lake system, the removal of organic pollutants in sediments using zero-valent iron $\left(\mathrm{Fe}^{0}\right)$ for in-situ sediment treatment was studied. The effects of zero valent iron $\left(\mathrm{Fe}^{0}\right)$ on the overlying water including DO, $\mathrm{Eh}, \mathrm{pH}$ and $\mathrm{COD}_{\mathrm{Cr}}$ were investigated and the effects on the sediments including the number and kinds of organic pollutants, total organic content were discussed. The results showed that with adding $\mathrm{Fe}^{0}$ for treating 80 days, (1) the amount of low-molecularweight organics ( of below 200) were increasing apparently which could be easy to degrade detecting by GC-MS. The removal rate of organic content in sediments was around 44\%. (2) the value of $\mathrm{pH}$ was rising to 8.4 , while the Eh was declining from $150 \mathrm{mV}$ to 74 $\mathrm{mV}$ and the DO concentration of overlying water depleted dramatically from $6.6 \mathrm{mg} / \mathrm{L}$ to $0.2 \mathrm{mg} / \mathrm{L}$. The system formed an anaerobic environment. $\mathrm{COD}_{\mathrm{Cr}}$ concentration of overlying water was lower than that in pure bottom-water system. In addition, there were certain relations among $\mathrm{DO}, \mathrm{Eh}, \mathrm{pH}$ and $\mathrm{COD}_{\mathrm{Cr}}$ in overlying water. In conclusion, the removal of organic pollutants was effective by adding $\mathrm{Fe}^{0}$ in the sediments, and this effect on overlying water quality was not permanent and seriously.
\end{abstract}

Keywords: In-situ sediment treatment; zero-valent iron $\left(\mathrm{Fe}^{0}\right)$; sediment; organic pollutants; overlying water

底泥有机污染是湖泊污染控制亟待解决的突出问题之一. 湖泊底泥中蓄积了大量因大气沉降、雨水淋 溶冲刷、废水排放等产生的有机污染物,这些污染物会在适当条件下与上覆水体发生物理、化学和生物作用

* 国家水体污染控制与治理科技重大专项(2017ZX07301002-05) 和国家自然科学基金项目 $(21467014,21767018)$ 联 合资助. 2017-06-09 收稿;2017-09-06 收修改稿. 章萍(1981 ), 女, 博士, 副研究员;E-mail: zhangping@ ncu.edu. cn. 
而被重新释放回上覆水体, 成为影响和制约湖泊上覆水质的主要二次污染源 ${ }^{[1-2]}$. 底泥原位修复技术因具有 投资少、易操作、对生态系统干扰小等优点, 在底泥污染修复技术上备受关注 ${ }^{[3]}$. 零价铁 $\left(\mathrm{Fe}^{0}\right)$ 在修复土壤和 地下水污染方面已得到广泛应用 ${ }^{[4-5]}$, 而向底泥投加 $\mathrm{Fe}^{0}$, 借助其还原、电解、混凝吸附等化学、物理作用达到 有效降解或消除有机污染的原位药剂投加法是近年来提出的一种新方法. 由于其具有修复周期短、处理成 本低且不易造成二次污染等优势, 已成为底泥原位修复技术的研究热点 ${ }^{[6-7]}$. Sneath 等 $^{[6]}$ 考察 $\mathrm{Fe}^{0}$ 对底泥中 菲的降解效果, 结果表明投加 $5 \%$ 的 $\mathrm{Fe}^{0}$ 在 $56 \mathrm{~d}$ 后对菲的降解可达 $82 \%$; Wang 等 ${ }^{[8]}$ 研究不同质量的铁粉对 底泥中 1,3-二氯苯降解效果时发现, 添加 $2 \%$ 的还原铁粉在 $20 \mathrm{~d}$ 就可降解 $72.13 \%$ 的 1, 3-二氯苯. 然而, 目前 国内外相关研究均采用有机物加标的方式, 尚未见 有关 $\mathrm{Fe}^{0}$ 在实际底泥体系下对有机物的降解效果及 其对上覆水体水质影响的研究.

因此,本研究基于前期对苏州河底泥有机污染 物分布的研究结果 ${ }^{[9]}$, 选取昌化路段的底泥和上覆 水体作为研究体系 (图 1), 在实验室模拟静态湖泊 体系条件下, 通过 GC-MS 以及常规水质检测等分析 手段,考察投加 $\mathrm{Fe}^{0}$ 前后实际底泥中有机物数量、种 类、含量以及上覆水体水质 ( 如溶解氧 (DO) 浓度、 氧化还原电位 $(\mathrm{Eh}) 、$ 化学需氧量 $\left(\mathrm{COD}_{\mathrm{Cr}}\right) 、 \mathrm{Fe}^{3+}$ / $\mathrm{Fe}^{2+}$ ) 变化情况, 以期为实际湖泊底泥治理提供理论 依据和技术支持.

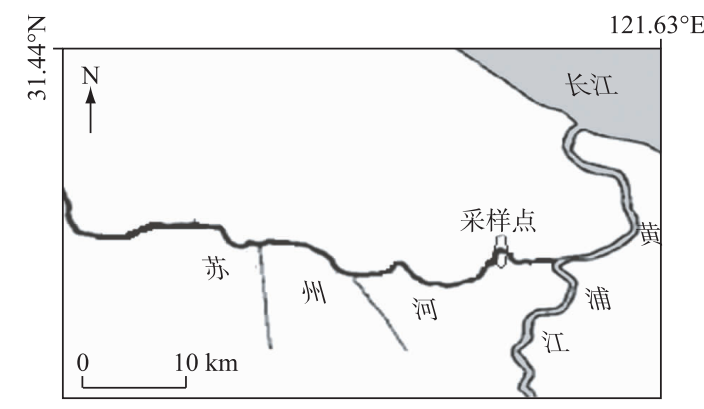

图 1 苏州河底泥采样断面分布

Fig.1 Distribution of sampling stations of sediment in Suzhou Creek

\section{1 材料与方法}

\section{1 实验材料}

1.1.1 底泥和上覆水 底泥利用抓斗式采泥器采集 (表层约 $10 \mathrm{~cm}$ ), 去除碎石等杂物后密封, 静置沥去水分; 水样为该断面 $15 \sim 30 \mathrm{~cm}$ 的表层水, 两者均保存于 $4^{\circ} \mathrm{C}$ 冰箱备用. 底泥与上覆水的理化性质见表 1 . 此外, 涉 及有机物分析的底泥在 $-20^{\circ} \mathrm{C}$ 中冷冻保存.

表 1 实验底泥与上覆水的主要理化性质

Tab.1 Main physical and chemical properties of tested samples

\begin{tabular}{cccccc}
\hline 样品 & 含水率 $/ \%$ & $\mathrm{pH}$ & $\mathrm{ORP} / \mathrm{mV}$ & $\mathrm{DO} /(\mathrm{mg} / \mathrm{L})$ & $\mathrm{COD}_{\mathrm{Cr}} /(\mathrm{mg} / \mathrm{kg})$ \\
\hline 底泥 & 53.64 & 7.24 & -153 & - & 36659 \\
上覆水 & - & 7.32 & 157 & 6.6 & 71.34 \\
\hline
\end{tabular}

1.1.2 实验药剂 $\mathrm{Fe}^{0}$ 为经酸洗进行预处理过的还原铁粉 (购于上海泰恒科学仪器有限公司) ; 邻苯二甲酸丁 脂、丙酮, 色谱纯; 正已烷、二氯甲烷, 分析纯, 经二次蒸馏后使用; 无水硫酸钠, 分析纯, $450^{\circ} \mathrm{C}$ 灼烧 $4 \mathrm{~h}$ 后待 用; 硅胶和中性氧化铝经二氯甲烷索氏抽提 $72 \mathrm{~h}$ 后于 120 和 $180^{\circ} \mathrm{C}$ 分别活化 $12 \mathrm{~h}$, 冷却平衡 $12 \mathrm{~h}$, 加人 $3 \%$ 的 去离子水活化, 平衡 $12 \mathrm{~h}$ 后浸于正已烷中待用.

\section{2 实验方法}

称取摚拌均匀后的底泥样品 $2000 \mathrm{~g}$, 平铺于底径为 $25 \mathrm{~cm}$ 的塑料容器中, 考虑药剂投加量过多会对底泥 及上覆水体有一定的影响 ${ }^{[10]}$, 称取底泥总重 $5 \%$ 的铁粉, 均匀撒于底泥表面, 彻底摚拌至均匀混合. 为模拟 苏州河实际体系, 即按照泥水高度比约为 $1: 6$ 的比例 ${ }^{[11]}$, 将约 $6000 \mathrm{ml}$ 水样以虹吸方式加人桶中, 加盖密封, 放置于人工恒温气候室 $\left(25^{\circ} \mathrm{C}\right)$ 中静置 $24 \mathrm{~h}$ 后, 每隔 $10 \mathrm{~d}$ 取样监测底泥 $\mathrm{COD}_{\mathrm{Cr}}$ 及上覆水的 $\mathrm{DO} 、 \mathrm{Eh} 、 \mathrm{pH} 、 \mathrm{COD}_{\mathrm{Gr}}$ 等指标. 在相同条件下以纯底泥一水体系作为空白对照, 处理周期设定为 $80 \mathrm{~d}$, 实验中均设 3 个平行样测定. 此外, 反应 $80 \mathrm{~d}$ 前后, 采用 GC-MS 分析手段对 $\mathrm{Fe}^{0}$ 处理前后底泥有机物数量和种类的变化情况进行考察.

\section{3 分析方法}

1.3.1 底泥有机物测定 样品前处理: 将冷冻保存的样品取出, 经冷冻干燥、研磨过篮后, 称取一定量样品分 
别进行有机物分析. 用二氯甲烷抽提, 收集抽提液. 抽提液经旋转蒸发浓缩, 转换溶剂, 并浓缩至 $1 \mathrm{ml}$. 浓缩 液经分离, 并选取不同极性有机溶剂分次洗脱, 收集目标化合物, 再经旋转蒸发并在氮气氛围下对其定容. 加人内标六甲基苯, 以备 GC-MS 分析.

GC-MS 分析条件: GC-MS 所用毛细管色谱柱为 DB-5MS ( $30 \mathrm{~m} \times 0.25 \mathrm{~mm} \times 0.25 \mu \mathrm{m})$ ), 载气为 $\mathrm{He}$ (纯度 $99.999 \%$ ), 载气流速为 $1.0 \mathrm{ml} / \mathrm{min}$; 程序升温为初温 $50^{\circ} \mathrm{C}$ (保留 $2 \mathrm{~min}$ ), 以 $10^{\circ} \mathrm{C} / \mathrm{min}$ 升温至 $280^{\circ} \mathrm{C}$ (保留 10 $\mathrm{min}$ ), 再以 $5^{\circ} \mathrm{C} / \mathrm{min}$ 升温至 $300^{\circ} \mathrm{C}$ (保留 $2 \mathrm{~min}$ ); MS 检测条件为电子轰击源电离电压 $70 \mathrm{eV}$, 离子源温度 $220^{\circ} \mathrm{C}$, 接口温度 $270^{\circ} \mathrm{C}$.

质量保证/质量控制 $(\mathrm{QA} / \mathrm{QC})$ : 样品抽提前, 向每个分析样品中加人回收率指示物, 用于监测分析过程 对目标化合物的影响, 确定样品回收率.

底泥有机质含量采用重铬酸钾油浴加热方法测定, $\mathrm{COD}_{\mathrm{Cr}}$ 含量采用密封恒温法测定 ${ }^{[12]}$.

1.3.2 上覆水体 DO 浓度 用 HI9142 式携带式溶解氧测定仪测定, Eh 用 5041 型笔式 ORP 计测定, $\mathrm{pH}$ 值用 PHS-3C $\mathrm{pH}$ 计测定, $\mathrm{Fe}^{3+}$ 还原为 $\mathrm{Fe}^{2+}$, 采用菲咯嗪分光光度法测定 $(562 \mathrm{~nm})^{[13]}$, 上覆水体 $\mathrm{COD}_{\mathrm{Cr}}$ 浓度采用重 铬酸钾法测定.

\section{2 结果与讨论}

\section{$2.1 \mathrm{Fe}^{0}$ 对底泥有机物的降解效果}

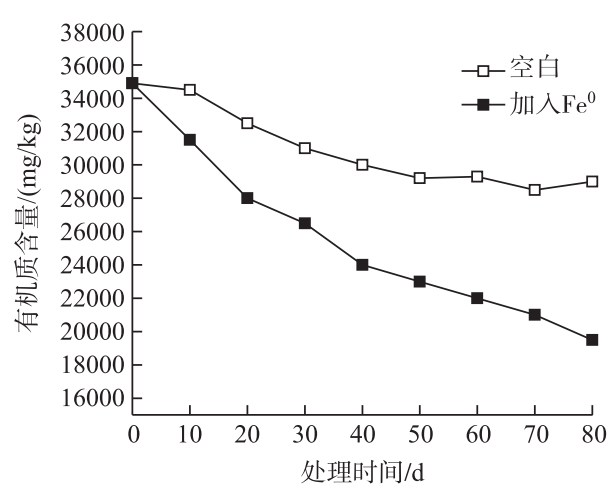

图 2 投加 $\mathrm{Fe}^{0}$ 对底泥有机质含量的影响

Fig. 2 The effects of organic content in sediment after adding the zero valent iron
2.1.1 底泥有机质含量变化 由图 2 可见, 恒温下 $\left(25^{\circ} \mathrm{C}\right)$, 无论纯底泥一水体系还是投加 $\mathrm{Fe}^{0}$ 体系, 底泥总有机质含量 均呈下降趋势. 在纯底泥一水体系中, 底泥总有机质含量在 $80 \mathrm{~d}$ 内下降缓慢, 由 $34720 \mathrm{mg} / \mathrm{kg}$ 降至 $29050 \mathrm{mg} / \mathrm{kg}$, 去除 率仅为 $16 \%$. 从结果上看, 底泥有机物自身存在一定的降 解, 这主要由微生物代谢作用所致 ${ }^{[14]}$. 而底泥中投加 $\mathrm{Fe}^{0}$ 后, 其有机质含量在前 $40 \mathrm{~d}$ 由 $34720 \mathrm{mg} / \mathrm{kg}$ 下降至 24000 $\mathrm{mg} / \mathrm{kg}$, 去除率已达 $31 \%$, 而随后的 $40 \mathrm{~d}$, 下降速率较前期 变缓, 底泥有机质含量总去除率为 $44 \%$, 明显高于纯底泥一 水体系, 说明 $\mathrm{Fe}^{0}$ 可有效提高底泥有机物的去除效率. $\mathrm{Fe}^{0}$ 具有还原性, 能有效降解有机物, 但随着其表面逐渐钝化, 会导致反应后期还原性降低, 对有机物去除效果下 降 ${ }^{[5-16]}$. 因此, 从结果上可以看出, $\mathrm{Fe}^{0}$ 作用底泥有机物的 最优时间主要集中在前 $40 \mathrm{~d}$.

2.1.2 底泥有机物数量及种类的变化为进一步反映 $\mathrm{Fe}^{0}$

对底泥中有机物的降解过程, 本研究通过 GC-MS 分析手段考察 $\mathrm{Fe}^{0}$ 处理前后底泥有机物数量和种类上的变 化情况. 从图 3 看出, $\mathrm{Fe}^{0}$ 处理 $80 \mathrm{~d}$ 后底泥中有机物在数量比原底泥体系明显增多; 在保留时间 $20 \mathrm{~min}$ 之前, 出现了分子量低于 200 的原底泥中未检测出的有机物 (如保留时间 8.20、16.69、18.96 min 等). 结合表 2 可 以看出, 原底泥中有机物多为长链大分子有机物, 如十六酸、十六醇、二氢胆固醇等, 同时还检出大量苯环类 有机物, 如对位壬基苯酚、邻苯二甲酸二 (2-乙基乙酯) 等. 而经 $\mathrm{Fe}^{0}$ 处理后的底泥有机物, 如乙二烯、丁烯二 酸、苯甲酸等, 均为分子结构简单、最多含有一个苯环并出现了双键结构的有机物. 肖利平等报道 $\mathrm{Fe}^{0}$ 在还原 过程中, 可将有机物中的苯环结构打开, 使其变成单双键交替的共轭链结构, 还可将共轭结构的有机物继续 还原破坏, 使有机物结构发生变化, 转化为小分子有机物 ${ }^{[17]}$. 由此看出, 底泥中投加 $\mathrm{Fe}^{0}$ 后, 含苯环结构等难 降解有机物在 $\mathrm{Fe}^{0}$ 还原性作用下结构发生变化, 转化为易分解的小分子有机物, 从而促进有机物的进一步 降解.

\section{$2.2 \mathrm{Fe}^{0}$ 对上覆水体水质的影响}

2.2.1 DO 浓度和 $\mathrm{Eh}$ 的变化 从图 4 可以看出, 恒温下 $\left(25^{\circ} \mathrm{C}\right)$, 无论是纯底泥一水体系, 还是投加 $\mathrm{Fe}^{0}$ 后, 上 覆水体的各个物化参数变化趋势都是一致的: DO 浓度和 Eh 随着时间均呈递降趋势. 与纯底泥一水体系相比, 
$\mathrm{Fe}^{0}$ 体系中上覆水体的 DO 浓度和 $\mathrm{Eh}$ 的降幅均偏大, 并且在前 $40 \mathrm{~d}$ 内变化趋势尤为显著. 对于纯底泥一水体 系来说, 由于底泥微生物在降解有机物过程中需要大量上覆水体的 DO, 而实验体系为封闭体系, 致使上覆 水体中 DO 不断被消耗而呈递降趋势; 而 $\mathrm{Fe}^{0}$ 作为还原性物质, 与体系 DO 接触后发生反应, 快速消耗 DO, 致 使上覆水体 DO 浓度在短时间内比纯底泥一水体系下降得更快, 进而促使上覆水体的氧化能力减弱 (即 Eh 下降). 上覆水体的 $\mathrm{DO}$ 浓度和 $\mathrm{Eh}$ 随时间变化与底泥 $\mathrm{COD}_{\mathrm{Cr}}$ 变化趋势较为一致, 进一步说明 $\mathrm{Fe}^{0}$ 对底泥有机 物有降解作用.

表 2 GC/MS 图谱中保留时间所对应的有机物

Tab.2 The organic matter corresponding to the retention time in the GC/MS spectrum

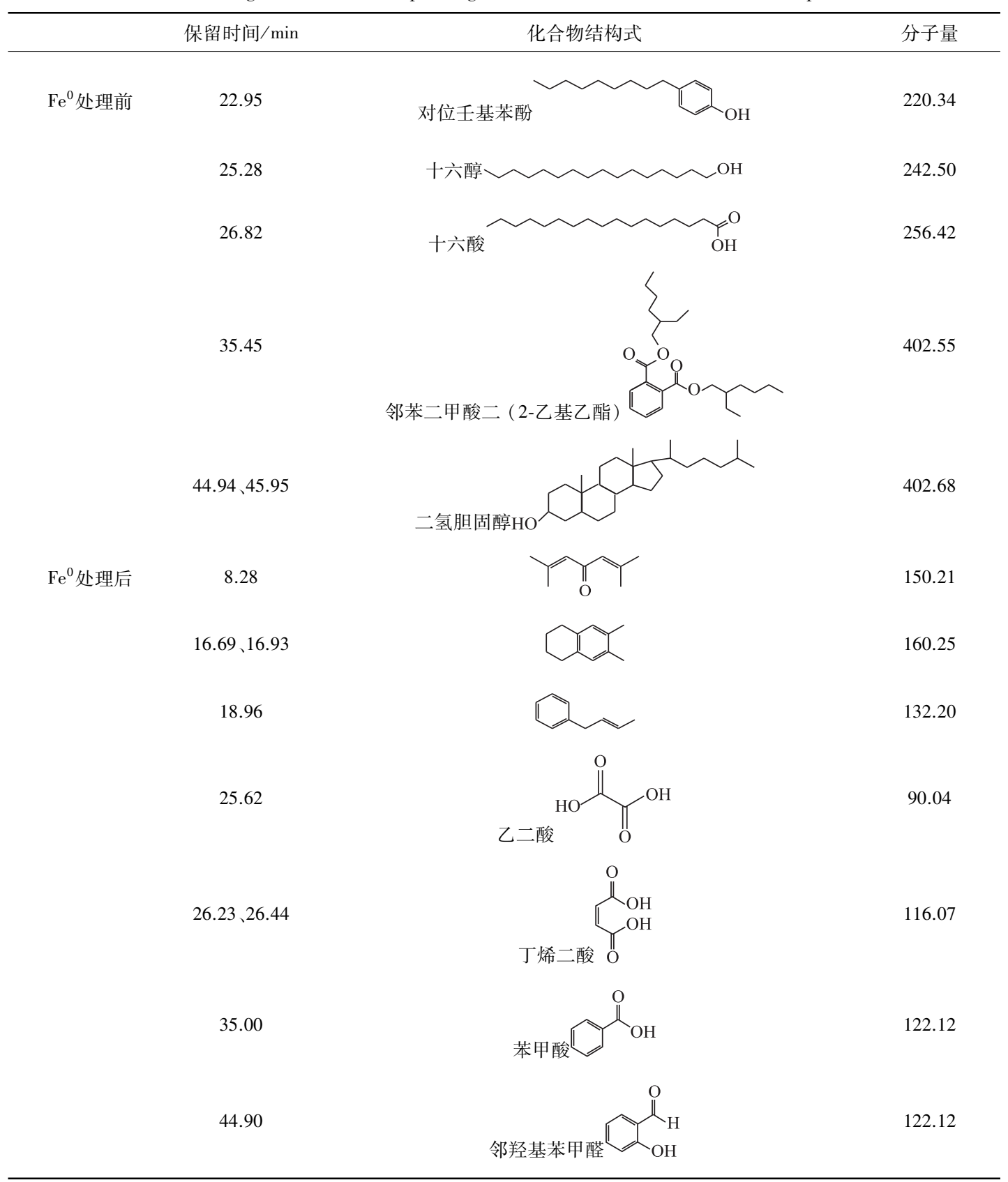



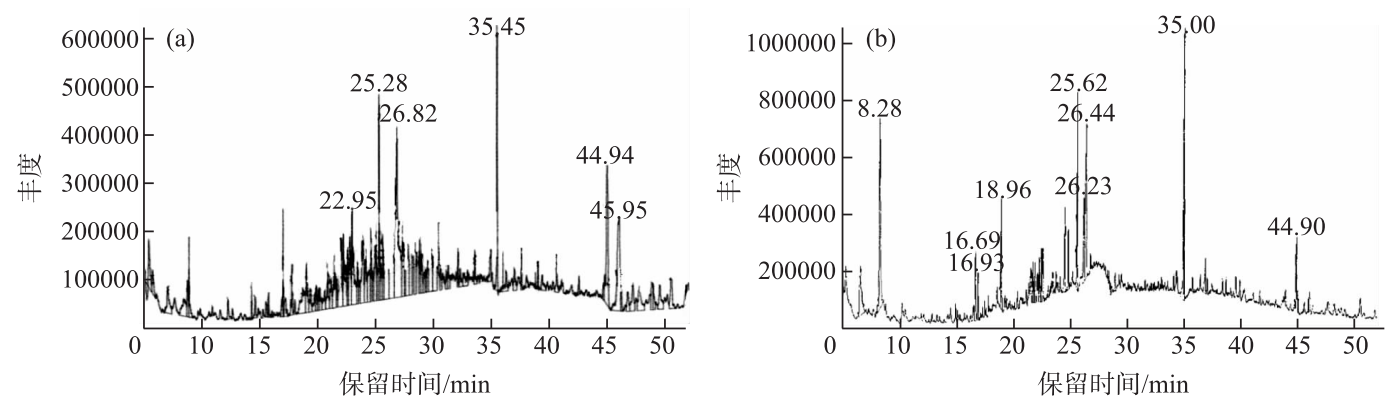

图 $3 \mathrm{Fe}^{0}$ 处理前 $(\mathrm{a})$ 和处理后 (b) 底泥 GC/MS 图谱

Fig.3 The GC/MS chromatography of the sediments before (a) and after (b) treated with zero valent iron
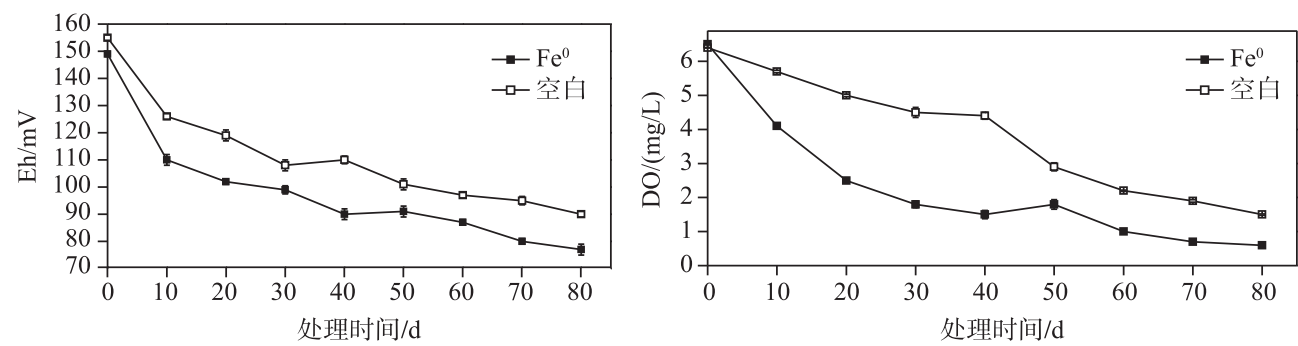

图 4 投加 $\mathrm{Fe}^{0}$ 反应前后上覆水中 $\mathrm{DO}$ 浓度和 $\mathrm{Eh}$ 的变化

Fig.4 The effect of zero valent iron on DO concentration and Eh in overwater system

2.2.2 $\mathrm{pH}$ 及 $\mathrm{Fe}^{3+} / \mathrm{Fe}^{2+}$ 的变化 $\mathrm{Fe}^{0}$ 降解有机物的过程主要是消耗体系 $\mathrm{H}^{+}$, 形成 $\mathrm{Fe}^{2+}$ 与有机物 (如卤烃) 发生 氧化还原反应, 使其脱氯或转化为小分子有机物, 同时生成 $\mathrm{Fe}^{3+}$, 其反应方程式为 ${ }^{[18]}$ :

$$
\begin{gathered}
\mathrm{Fe}+2 \mathrm{H}^{+} \rightarrow \mathrm{Fe}^{2+}+2[\mathrm{H}] \\
\mathrm{RCl}+2 \mathrm{Fe}^{2+}+\mathrm{H}^{+} \rightarrow \mathrm{RHCl}^{-}+2 \mathrm{Fe}^{3+}
\end{gathered}
$$

基于此, 本研究考察了恒温下 $\left(25^{\circ} \mathrm{C}\right)$, 投加 $\mathrm{Fe}^{0}$ 后上覆水体中 $\mathrm{pH}$ 及 $\mathrm{Fe}^{3+} / \mathrm{Fe}^{2+}$ 随时间的变化, 并以纯底 泥一水体系作为空白对照. 从图 5 可以看出, 投加 $\mathrm{Fe}^{0}$ 体系与纯底泥一水体系上覆水的 $\mathrm{pH}$ 值均呈上升趋势, 投加 $\mathrm{Fe}^{0}$ 后的上覆水 $\mathrm{pH}$ 明显高于空白. $\mathrm{Fe}^{0}$ 降解有机物过程中, 消耗底泥孔隙水 $\mathrm{H}^{+}$, 由于底泥与水界面的交 换扩散作用使得上覆水的 $\mathrm{H}^{+}$浓度降低, $\mathrm{pH}$ 值升高. 因此, 上覆水 $\mathrm{pH}$ 变化进一步证明了 $\mathrm{Fe}^{0}$ 在底泥体系与有 机物发生了氧化还原反应. 结合上覆水体 $\mathrm{Fe}^{3+} / \mathrm{Fe}^{2+}$ 随时间的变化趋势, 对于 $\mathrm{Fe}^{0}$ 体系, 水中 $\mathrm{Fe}^{3+} / \mathrm{Fe}^{2+}$ 比值在
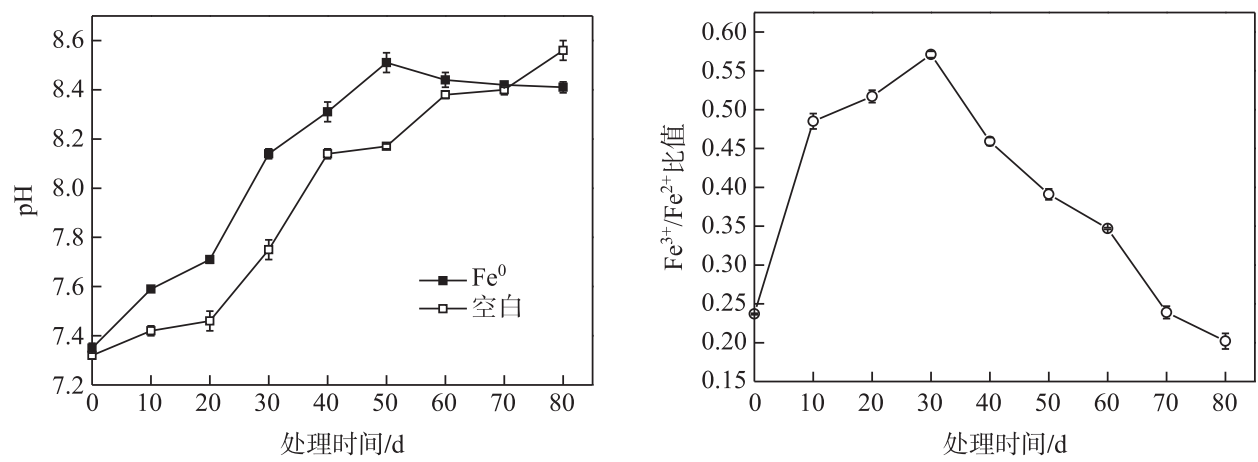

图 5 投加 $\mathrm{Fe}^{0}$ 反应前后上覆水 $\mathrm{pH}$ 和 $\mathrm{Fe}^{3+} / \mathrm{Fe}^{2+}$ 的变化

Fig.5 The effect of zero valent iron on $\mathrm{pH}$ and $\mathrm{Fe}^{3+} / \mathrm{Fe}^{2+}$ in overwater system 
前 $30 \mathrm{~d}$ 呈上升趋势, $\mathrm{Fe}^{3+}$ 浓度相对增加, 说明 $\mathrm{Fe}^{0}$ 在此段时间内具有一定的还原活性. $30 \mathrm{~d}$ 后 $\mathrm{Fe}^{3+} / \mathrm{Fe}^{2+}$ 呈下 降趋势, 说明 $\mathrm{Fe}^{0}$ 逐渐失去活性; 此外, 进人上覆水的 $\mathrm{Fe}^{3+} 、 \mathrm{Fe}^{2+}$ 与 $\mathrm{OH}^{-}$反应生成 $\mathrm{Fe}(\mathrm{OH})_{2}$ 和 $\mathrm{Fe}(\mathrm{OH})_{3}$, 吸附水 中部分有机物得以沉淀, 也促进 $\mathrm{Fe}^{3+} / \mathrm{Fe}^{2+}$ 的下降. 由上述结果看出, 投加 $\mathrm{Fe}^{0}$ 不会对上覆水体水质产生持久、 较大的影响.

2.2.3 $\mathrm{COD}_{\mathrm{Cr}}$ 浓度变化 恒温下 $\left(25^{\circ} \mathrm{C}\right)$, 对于 $\mathrm{Fe}^{0}$ 体系, 上 覆水 $\mathrm{COD}_{\mathrm{Gr}}$ 浓度在 $10 \mathrm{~d}$ 内迅速上升至 $95 \mathrm{mg} / \mathrm{L}$, 随后逐 步降低, 且最终低于空白体系. 结合前文讨论可知, 由于 反应初期 $\mathrm{Fe}^{0}$ 与底泥中有机物发生作用生成可溶性小分 子有机物通过间隙水扩散到水中, 才导致上覆水的 $\mathrm{COD}_{\mathrm{Cr}}$ 浓度急速增加; 但随后 $\mathrm{Fe}^{2+} 、 \mathrm{Fe}^{3+}$ 含量升高, 且形成 氢氧化物絮状沉淀吸附上覆水中有机物得以沉淀, 致使 最终上覆水中 $\mathrm{COD}_{\mathrm{Cr}}$ 浓度下降至空白对照体系以下 (图 6). 因此, 以上结果可进一步看出, $\mathrm{Fe}^{0}$ 作为底泥稳定化 化学药剂不会对上覆水造成持久性影响.

2.2.4 DO 浓度、 $\mathrm{Eh} 、 \mathrm{pH} 、 \mathrm{Fe}^{3+} / \mathrm{Fe}^{2+}$ 及 $\mathrm{COD}_{\mathrm{Cr}}$ 浓度的相关性

SPSS 软件可研究随机变量的相关关系, Pearson 相关 系数用于衡量定距变量间的线性关系, Spearman's rank 秩相关系数可描述两个变量之间的关联程度与方向. 目

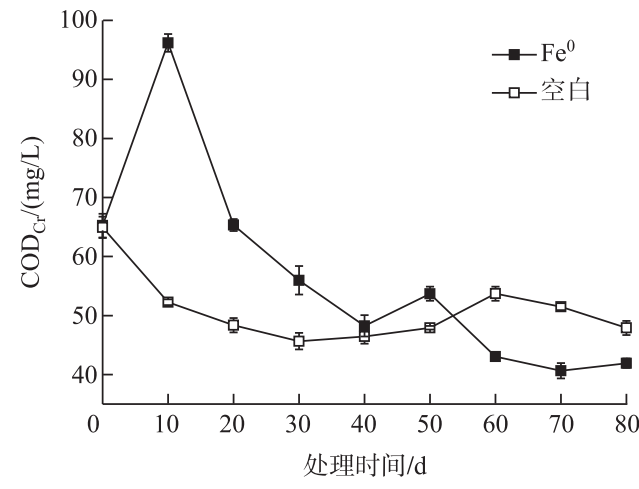

图 6 投加 $\mathrm{Fe}^{0}$ 反应前后上覆水 $\mathrm{COD}_{\mathrm{Cr}}$ 变化

Fig. 6 The effect of zero valent iron on $\mathrm{COD}_{\mathrm{Cr}}$ in overwater system 前, 三者结合被广泛用于水质指标相关性研究. 由图 4 6 可见, $\mathrm{Fe}^{0}$ 与有机污染物发生作用后, 产物通过底泥 一水界面交换作用, 使得上覆水 $\mathrm{DO}$ 浓度、 $\mathrm{Eh} 、 \mathrm{pH} 、 \mathrm{Fe}^{3+} / \mathrm{Fe}^{2+}$ 及 $\mathrm{COD}_{\mathrm{Cr}_{\mathrm{r}}}$ 农度均发生变化. 为确定 $\mathrm{DO}$ 浓度、 $\mathrm{Eh}$ 、 $\mathrm{pH} 、 \mathrm{Fe}^{3+} / \mathrm{Fe}^{2+}$ 和 $\mathrm{COD}_{\mathrm{Cr}}$ 浓度之间的相互关系, 本研究采用统计分析软件 SPSS 24.0 进行样本数据正态分布分 析以及 Pearson 相关系数和 Spearman's rank 相关系数分析. 经 K-S 检验法的计算结果见表 3. 数据分析结果 显示, 除投加 $\mathrm{Fe}^{0}$ 的上覆水体 $\mathrm{COD}_{\mathrm{Cr}}$ 浓度为非正态分布以外 $(P<0.05)$, 其他参数值均服从正态分布 $(P>$ $0.05)$. 对于投加 $\mathrm{Fe}^{0}$ 的体系, 上覆水体 $\mathrm{DO}$ 浓度、 $\mathrm{pH}$ 和 $\mathrm{Eh}$ 以及 $\mathrm{COD}_{\mathrm{Gr}}$ 浓度之间具有较显著的相关性 $(P<$ $0.01)$. 因此通过相关性计算结果看出, 为提高 $\mathrm{Fe}^{0}$ 对底泥有机物的降解能力, 减少对上覆水体的影响, 可通 过调节上覆水体中 DO 浓度、 $\mathrm{pH}$ 和 $\mathrm{Eh}$ 得以实现.

表 $3 \mathrm{Fe}^{0}$ 体系中上覆水 $\mathrm{DO} 、 \mathrm{Eh} 、 \mathrm{pH} 、 \mathrm{Fe}^{3+} / \mathrm{Fe}^{2+}$ 及 $\mathrm{COD}_{\mathrm{Cr}}$ 的相关系数

Tab.3 The correlation coefficient of DO, Eh, $\mathrm{pH}, \mathrm{Fe}^{3+} / \mathrm{Fe}^{2+}$ and $\mathrm{COD}_{\mathrm{Cr}}$ in the presence of zero valent iron in overwater system

\begin{tabular}{|c|c|c|c|c|c|}
\hline & DO & Eh & $\mathrm{pH}$ & $\mathrm{Fe}^{3+} / \mathrm{Fe}^{2+}$ & $\mathrm{COD}_{\mathrm{Cr}}$ \\
\hline DO & 1 & $0.980^{\mathrm{a} * *}$ & $-0.906^{\mathrm{a} * *}$ & & $0.920^{\mathrm{b} * *}$ \\
\hline Eh & & 1 & $-0.852^{\mathrm{a} * *}$ & & $0.922^{\mathrm{b} * *}$ \\
\hline $\mathrm{pH}$ & & & 1 & & $-0.801^{\mathrm{b} * *}$ \\
\hline \multicolumn{6}{|l|}{$\mathrm{Fe}^{3+} / \mathrm{Fe}^{2+}$} \\
\hline $\mathrm{COD}_{\mathrm{Cr}}$ & & & & & 1 \\
\hline
\end{tabular}

$\mathrm{a}$ 代表 Pearson 相关系数; b 代表 Spearman 相关系数; $*$ 代表 $\partial=0.05 ; * *$ 代表 $\partial=0.01$.

\section{3 结论}

1) 向底泥中投加 $\mathrm{Fe}^{0}$ 后, 底泥有机质含量的去除率可达 $44 \%$; 通过 GC-MS 分析结果看出, 经 $\mathrm{Fe}^{0}$ 处理后 的底泥中易降解的小分子有机物数量和种类明显增加.

2) 投加 $\mathrm{Fe}^{0}$ 体系上覆水中 $\mathrm{DO}$ 浓度和 $\mathrm{Eh}$ 随时间下降, 且低于纯底泥一水体系测定值; $\mathrm{pH}$ 呈上升趋势, 最 终稳定于 $8.4 ; \mathrm{COD}_{\mathrm{Cr}}$ 浓度先增加后降低, 低于纯底泥一水体系测定值. 上覆水中 $\mathrm{DO}$ 浓度、 $\mathrm{pH}$ 、 Eh 以及 $\mathrm{COD}_{\mathrm{Cr}}$ 浓度之间具有较显著的相关性. 
3 ) 底泥中 $\mathrm{Fe}^{0}$ 的投加不会对上覆水体水质产生持久、较大的影响, 底泥原位投加 $\mathrm{Fe}^{0}$ 法在底泥有机污染 物的治理中具有良好的应有前景.

\section{4 参考文献}

[ 1 ] Wang WW, Wang SH, Jiang X et al. Occurrence characteristics and release potential of nitrogen fractions in sediment of Lihu Lake. China Environmental Science, 2017, 37 (1) : 292-301. [王雯雯, 王书航, 姜霞等. 蟙湖沉积物不同形态氮 赋存特征及其释放潜力. 中国环境科学, 2017, 37(1): 292-301.]

[ 2 ] Han Q, Xue S, Liu Y et al. Release pathway and influencing factors of dissolved organic matter in river sediments. China Environmental Science, 2016, 36(12) : 3737-3749. [ 韩琦, 薛爽, 刘影等. 河流底泥中溶解性有机物的释放途径及 影响因素研究. 中国环境科学, 2016, 36(12): 3737-3749.]

[ 3 ] Song B, Zeng G, Gong J et al. Evaluation methods for assessing effectiveness of in situ remediation of soil and sediment contaminated with organic pollutants and heavy metals. Environment International, 2017, 105: 43.

[ 4 ] Cao M, Ye Y, Chen J et al. Remediation of arsenic contaminated soil by coupling oxalate washing with subsequent ZVI/ Air treatment. Chemosphere, 2015, 144: 1313.

[ 5 ] Huaping D, Ya C, Guodong S et al. The roles of a pillared bentonite on enhancing Se(VI) removal by ZVI and the influence of co-existing solutes in groundwater. Journal of Hazardous Materials, 2016, 304(3) : 306-312.

[ 6 ] Sneath HE, Hutchings TR, de Leij FA. Assessment of biochar and iron filing amendments for the remediation of a metal, arsenic and phenanthrene co-contaminated spoil. Environmental Pollution, 2013, 178(1) : 361-366.

[ 7 ] Ahn JY, Kim C, Kim HS et al. Effects of oxidants on in situ treatment of a DNAPL source by nanoscale zero-valent iron: A field study. Water Res, 2016, 107: 57-65.

[ 8 ] Wang XX, Zhang Y, Wang YF et al. Remediation of sediments contaminated with 1, 3-dichlorobenzene using zero-valent iron. Research of Environmental Sciences, 2009, 22(3) : 289-293. [王新新, 张颖, 王元芬. 零价铁修复 1,3-二氯苯污 染底泥. 环境科学研究, 2009, 22(3): 289-293.]

[ 9 ] Dong Y, Zou LP, Zhang XL. Preliminary study on the organics in top sediments of Suzhou Creek in Shanghai. Advances In Water Science, 2008, 19(4) : 494-499. [董煜, 邹联沛, 张晓岗. 苏州河上海市区段底泥有机物的初步研究. 水科学 进展, 2008, 19(4): 494-499.]

[10] Crane RA, Scott TB. Nanoscale zero-valent iron: future prospects for an emerging water treatment technology. Journal of Hazardous Materials, 2012, 211/212 : 112-125.

[11] Xu SY, Chen ZL et al eds. Pollution and treatment for sediment of Suzhou River. Beijng: Science Press, 2003: 1-90. [许 世远, 陈振楼等. 苏州河底泥污染与整治. 北京: 科学出版社, 2003: 1-90.]

[12] Zhang K, Li B, Wang DZ et al. Contaminant $\left(\mathrm{COD}_{\mathrm{Cr}}\right)$ release from river bottom sediments under flow conditions. Acta Scientiae Circumstantiae, 2010, 30(5): 985-989. [张坤, 李彬, 王道增. 动态水流条件下河流底泥污染物 $\left(\mathrm{COD}_{\mathrm{Cr}}\right)$ 释放研究. 环境科学学报, 2010, 30(5): 985-989.]

[13] El-Temsah YS, Sevcu A, Bobcikova K et al. DDT degradation efficiency and ecotoxicological effects of two types of nanosized zero-valent iron (nZVI) in water and soil. Chemosphere, 2016, 144: 2221-2228.

[14] Wang C, Xi JY, Hu HY et al. Comparision of microbial metabolic characteristics in UV-biofilter treatment process. Acta Scientiae Circumstantiae, 2010, 30(8) : 1587-1592. [王灿, 席劲瑛, 胡洪营等. 紫外-生物过滤联合工艺和单一生物 过滤工艺中微生物代谢特性的比较. 环境科学学报, 2010, 30(8): 1587-1592.]

[15] Zhou HY, Nie YZ, Chen Y et al. Effects of EDTA on the reductive dechlorination of 2,4-D by Pd/Fe. Environmental Science, 2016, 37(2) : 595-601. DOI: 10.13227/2016.02026. [周红艺, 聂亚中, 陈勇等. EDTA 对 $\mathrm{Pd} / \mathrm{Fe}$ 体系还原脱 氯 2,4-D 的影响. 环境科学, 2016, 37(2) : 595-601.]

[16] Zhou HY, Chen Y, Liang S et al. Catalytic dechlorination of 2,4-D in aqueous solution by nanoscale $\mathrm{Pd} / \mathrm{Fe}$ in the presence of EDTA. Acta Scientiae Circumstantiae, 2017, 37(2) : 671-679. [周红艺, 陈勇, 梁思等. EDTA 优化纳米 Pd/ $\mathrm{Fe}$ 对 2,4-D 的催化脱氯研究. 环境科学学报, 2017, 37(2) : 671-679.]

[17] Xiao LP, Guo Y, Deng ZY. Characteristics of direct black 19 decolorized by the bacterium Pseudomonas fluorescence and $\mathrm{Fe}^{0}$ integrated treatment system. Acta Scientiae Circumstantiae, 2013, 33(9): 2504-2510. [肖利平, 郭燕, 邓志毅等. $\mathrm{Fe}^{0}$ /苂光假单胞菌联合处理直接耐晒黑的脱色特性. 环境科学学报, 2013, 33(9): 2504-2510.]

[18] Ardo F, Santos A, Romero A et al. Fate of iron and polycyclic aromatic hydrocarbons during the remediation of a contaminated soil using iron-activated persulfate: A column study. Sci Total Environ, 2016, 566/567: 480-488. 